\title{
Informationsgehalt und Nutzen der Endosonographie
}

\author{
G. Feifel und U. Hildebrandt
}

Abteilung für Allgemeine Chirurgie, Abdominal- und Gefäßchirurgie (Direktor: Prof. Dr. G. Feifel), Chirurgische Universitäts-Klinik, W-6650 Homburg/Saar, Bundesrepublik Deutschland

Wenige neue diagnostische Verfahren haben in relativ kurzer Zeit eine solche Akzeptanz in der Chirurgie erlebt wie sonographische und endosonographische Untersuchungsmethoden $[5,9]$. Aufbauend auf den ersten Studien von Wild in den 50er Jahren erfolgte im letzten Jahrzehnt eine enorme technologische Entwicklung der Endosonographie und ihre breite Anwendung in der Urologie, Gynäkologie und Gastroenterologie [2, 3, 6, 7, $10-12]$.

Im Mittelpunkt der endosonographischen Diagnostik steht die präoperative Stadienbestimmung von Tumoren des oberen und unteren Verdauungstraktes. Durch in vitro- und in vivo-Untersuchungen wurden die Grundlagen für die sonographische Differenzierung des anatomischen Aufbaus der Darmwand geschaffen [1, 10]. In großer Übereinstimmung mit zahlreichen Untersuchern läßt sich feststellen, daß die Endosonographie bei der Darstellung der Darmwandschichten allen anderen Untersuchungsmethoden überlegen ist. Die hohe Aussagekraft der Endosonographie, gemessen an Sensitivität und Spezifität, liegt dabei speziell bei der Abklärung kleiner Tumore. Diese Feststellung allein, so erfreulich sie ist, genügt jedoch nicht um die Frage nach der klinischen Relevanz der Endosonographie zu beantworten. Ein hoher Informationsgehalt eines diagnostischen Verfahrens ist per se noch kein Beweis seines Nutzens für den $\mathrm{Pa}$ tienten [8]. Als Standard in der Beurteilung der Nützlichkeit neuer bildgebender Verfahren werden üblicherweise 3 Stufen unterschieden [8]:

1. Änderung des diagnostischen Vorgehens aufgrund des verbesserten Informationsgehaltes durch die neue Methode;

2. Änderung des Therapiekonzeptes aufgrund der verbesserten Information;

3. Positive Auswirkung des Diagnoseverfahrens auf den Krankheitsverlauf.

Alle bislang vorgelegten Daten zur Endosonographie belegen nur die verbesserte präoperative Information im Hinblick auf die Erkennung der Tumorpenetrationstiefe. Unbefriedigend bleibt die Beurteilung der Lymphknoten
[3]. Mit der gegenwärtigen apparativen Ausrüstung liegt die Grenze der Erkennbarkeit von Lymphknoten bei $3 \mathrm{~mm}$ Durchmesser. Ab einer Größe von $4 \mathrm{~mm}$ können Lymphknoten endosonographisch zuverlässig nachgewiesen werden. Eine sonographische Differenzierung bezüglich der Dignität von Lymphknoten ist zur Zeit nicht mit der nötigen Sicherheit möglich. Ausgehend von den Beobachtungen von Tio [10] wird die Echogenität der dargestellten Lymphknoten empirisch zur Dignitätsbeurteilung herangezogen. Echoreiche Lymphknoten werden als unspezifisch entzündlich, echoarme als malignitätsverdächtig klassifiziert. Es ist nicht verwunderlich, daß auf dieser relativ unscharfen Grundlage vollständig metastatisch befallene Lymphknoten zwar gut erkannt werden, eine beginnende Infiltration jedoch übersehen werden kann. Außerdem gibt es so gut wie keine klinischen Informationen über die Beeinflussung der Schallphänomene durch verschiedene Tumorarten.

Die zunehmende Zahl an endosonographischen Beobachtungen von Lymphknoten kann deshalb nicht darüber hinwegtäuschen, daß weitere systematische Untersuchungen am oberen und unteren Verdauungstrakt fehlen. Für die Beurteilung endoluminaler Ultraschallbefunde ist vor allem die Kenntnis des zu erwartenden normalen Lymphknotenstatus (Zahl, Größe, Lage) notwendig. Darüber hinaus müssen quantitative Daten über Metastasen in Lymphknoten verschiedener Größe erarbeitet werden, wie sie zum Beispiel von Giedel et al. vorgelegt wurden [4]. Parallel hierzu sind die methodischen Grenzen der sonographischen Lymphknotendiagnostik besser zu definieren. Es gilt unter anderem der Frage nachzugehen, ob die Schallschwächung ein ausreichendes Kriterium zur Erkennung maligner Veränderungen ist oder ob nicht vielmehr andere physikalische Parameter zur Differenzierung besser geeignet sind. In diesen Zusammenhang gehört auch das Problem frühe maligne Veränderungen in Adenomen endosonographisch zu erfassen. Die hierüber mitgeteilten Ergebnisse lassen eine generelle Bewertung noch nicht zu. Es erscheint deshalb ratsam, Sensitivität und Spezifität der Methode bei Adenom und Karzinom getrennt zu erheben. Schließlich bedürfen die 
im T-Stadium unterbewerteten Tumore einer besonders sorgfältigen Analyse, da sie unter Umständen AnlaB für therapeutische Fehlentscheidungen sind.

Vor dem Hintergrund dieser offenen Fragen läßt sich feststellen, da $B$ das diagnostische Potential der endoluminalen Sonographie noch nicht ausgeschöpft ist. Folglich lassen sich zum gegenwärtigen Zeitpunkt die ableitbaren therapeutischen Konsequenzen, mit Ausnahme rektaler Frühkarzinome, noch nicht hinreichend sicher definieren. Andererseits ist der Informationsgehalt der prä- und posttherapeutischen Endosonographie bereits jetzt so hoch, daB sie - vor allem beim Ösophaguskarzinom zum festen Bestandteil der Diagnostik gehört. Auch bei Berücksichtigung der noch unbefriedigenden Lymphknotenbeurteilung darf nicht vergessen werden, daß das Informationsdefizit ohne Endosonographie unvergleichlich größer war. Alle Erfahrungen deuten darauf hin, daB die Endosonographie in absehbarer Zeit zu einem wichtigen Instrument der Therapieplanung bei Eingriffen am oberen und unteren Verdauungstrakt wird. Voraussetzung ist allerdings, daß, parallel zur Optimierung der endosonographischen Information, therapeutische Alternativen für die jeweiligen Tumorstadien formuliert werden. Die Möglichkeit, daß die endosonographische Diagnostik vom Operateur selbst eingesetzt wird, kann nicht hoch genug bewertet werden. Diese Chance bedeutet aber auch eine wesentlich höhere Verantwortung. Der Nutzen dieser eleganten Diagnostik wird deshalb nicht ohne intensives Einüben und Einsehen und nicht ohne sorgfältige Befunddokumentation zu erreichen sein.

\section{Literatur}

1. Boscaini $M$ (1989) Lower gastrointestinal endoultrasound. Surg Endosc 3:29-32

2. Dragstedt J, Gammelgaard J (1983) Endoluminal ultrasonic scanning in the evaluation of rectal cancer: a preliminary report of 13 cases. Gastrointest Radiol 8:367-369

3. Feifel G, Hildebrandt U, Mortensen NJMcC (1990) Endosonography in gastroenterology, gynecology and urology. Springer, Berlin Heidelberg New York

4. Giedl J, Dworak O (1989) Anatomische Grenzen der Endosonographie im Gastrointestinaltrakt aus der Sicht des Pathologen. Endoskopie Heute 3:44

5. Hill CR (1986) Physical principles of medical ultrasonics. Ellis Horwood Limited, Halsted Press: a division of John Wiley and Sons, New York Chichester Brisbane Toronto

6. Lutz H, Rösch W (1976) Transgastroscopic ultrasonography. Endoscopy 8:203

7. Mortensen NJMcC (1986) Endosonography of the rectum (Symposium). Int Colorectal Dis 1:201-226

8. Ohmann Ch, Lorenz W (1986) Allgemeine Grundlagen klinischer Studien über diagnostische Verfahren. In: Hermanek $P$ (Hrsg) Bildgebende Verfahren in der Onkologie. Springer, Berlin Heidelberg New York, S 98-108

9. Siegel B (1988) Operative ultrasonography. Raven Press, New York

10. Tio ThL (1988) Endosonography in gastroenterology. Springer, Berlin Heidelberg New York

11. Wild JJ, Reid JM (1956) Diagnostic use of ultrasound. Br J Phys Med 11:248-264

12. Ziegler K (1990) Endosonographie, Technik und klinische Anwendung. Perimed, Erlangen 